\title{
Solution of the Rodriguez Equation in Modeling Volumetric Geometric Accuracy of Multi-Coordinate Systems
}

\author{
M.M. Stebulyanin", Ya.I. Pimushkin \\ Moscow State Technological University "STANKIN", RU-127055, Moscow, Russia
}

\begin{abstract}
The article describes the solution to the Rodrigues equation for determining the volumetric accuracy of multi-axis CNC-controlled systems. An algorithm for calculating the position of the axis of a rotary kinematic pair in problems of volumetric accuracy of mechanical motion of a portal-type system with an additional pair of rotation. The algorithm is based on the analytical solution of the Rodrigues equation in the inverse problem of finding the vector of the final rotation of the known modulus from the known initial and final values of the characteristic vector of the rotated rigid body. In contrast to the well-known direct problem, where based on a finite rotation vector known in direction and magnitude, and the initial value of the characteristic vector of a body, its final value is found, the inverse problem of the Rodrigues equation is not that common due to the nonlinearity and need to solve a nonlinear coupled system of second order equations. The results of this work make it possible to expand the dimension of the space of generalized coordinates of the system analyzed for the volumetric accuracy from three to four. This is expected contribute to the development of ultra-precise systems of controlled mechanical movement. The analytical results of this study were verified by comparing with numerical solutions of the inverse problem in Maple.
\end{abstract}

\section{Introduction}

Multi-axis systems with software-controlled movement of units and mechanisms are a an important part of modern mechanical engineering, in terms of both the technological equipment (machine tools, industrial robots) and measuring technologies (coordinate measuring machines). Highly efficient use of such systems at the moment is no longer possible without a systemic increase in the volumetric accuracy (VA) of the actuator equipment, that reflects the ability to accurately reproduce complex three-dimensional surfaces with a measuring tip or cutting edge of a tool [1-3].

For the first time, the concept of volumetric accuracy was introduced in the work of P.A. McKeown, J. Loxham [4], and, in the last decade, the problem of increasing VA has become widespread [5, 6]. In Russia, this concept was introduced in the work of Serkov [7]. Quantitatively, the volumetric accuracy is characterized by the volumetric error (VE), which for each point of the machining workspace is a vector directed from the specified (nominal) position of a certain pole of the tool to its actual position. To study the volumetric accuracy, many methods and approaches such as the DenavitHartenberg method [8], the method for describing the kinematics of a rigid body (rigid body kinematics) [9], the kinematics of a set of rigid bodies (Multiple-body kinematics) [10] and methods of matrix simulation [11] are used:. In ref. [12-13] articles, not only systematic but also random characteristics of equipment accuracy are considered
The ultimate goal of the study of volumetric accuracy is the construction of algorithms for correcting the real motion of a mechanical system in a generalized space relative to another, considered under the condition of an "ideal" arrangement of the axes of the kinematic pairs of the system. At the same time, it is known that, for example, with gantry three-coordinate kinematics, the axes of the telescopic pairs of the machine tool do not form an ideal orthogonal trihedron, and this discrepancy increases with the wear of the equipment.

\section{Results and discussion}

To correct the errors of the three-dimensional portal motion caused by this discrepancy, it is necessary to know the true position of the physical axes of translational displacements. The task becomes more complicated if the fourth rotational axis is included in the generalized space. In this case, it is not possible to correct motion errors by using only translational motion along the axes of the portal kinematic scheme. To solve the problem of motion correction, here it is also necessary to know the true position in space of the axis of rotation of the fourth kinematic pair.

Let explain what has been said in Figure 1, which conventionally shows a mechanism with three translational motions in a stationary Cartesian coordinate system and a fourth link, which is a rotor in a kinematic pair of a rotational type, with a stator connected to a coordinate system co-directed with the system. Translational linear movements are carried out along the

e-mail: mmsteb@rambler.ru 
physical axes of a generally non-Cartesian system with a common origin at point $\mathrm{O}$, which, due to design inaccuracies, does not coincide with the origin of the system. The 3x1 vector of generalized coordinates of linear displacements will be denoted by.

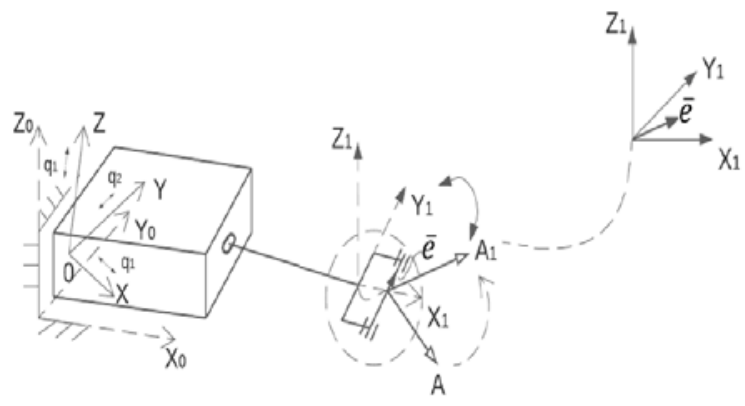

Fig. 1. Deviation of the physical axis of rotation from the ideal position

When turning about the real axis of the pair, the direction of which is set by the unit vector, the effector link moves to a position that can be obtained in the system, for example, on the basis of the theory of finite turns. Why it is necessary to obtain the radius vector of the effector pole in a stationary system, you can use the equation:

$$
\bar{r}_{A}=\bar{r}_{1}+\boldsymbol{C} \bar{q}+\bar{r}_{a},
$$

where $\mathrm{C}$ - constant $3 \mathrm{x} 3$ is the calibration matrix obtained on the basis of the calibration procedure of translational (linear) movements in the physical axes of telescopic pairs;

$\bar{r}_{1}$ - radius vector of the origin of the Cartesian system in the Cartesian system effector

$\bar{r}_{a}$ - radius vector in coordinate system

In the calibration procedure, which is known in principle and therefore is not considered in this work, the values and are obtained. There remains the problem of calculating a vector that completely depends on the true direction of the unit vector of the axis of the rotational kinematic pair.

The true direction of this axis, although it differs from the nominal one by an unknown value, remains unchanged and does not depend on the movement in telescopic kinematic pairs, which makes it possible to determine its unit vector $\mathrm{e}^{-}$.

\section{Rodrigues' rotation formula}

To find the real position of the axis of a rotational pair, as will be shown below, it is possible to use the Rodrigues' rotation formula:

$$
\overline{\rho^{\prime}}=\bar{\rho}+\frac{1}{1+\frac{1}{4}|\bar{\theta}|^{2}} \bar{\theta} \times\left(\bar{\rho}+\frac{1}{2} \bar{\theta} \times \bar{\rho}\right)
$$

$\bar{\rho}-$ radius vector of the initial position of the effector pole; $\overline{\rho^{\prime}}-\bullet$ radius vector of its final position;

$\bar{\theta}=2 \operatorname{tg} \frac{\chi}{2} \overline{\mathrm{e}}$, где $\chi$ - угол поворота вектора $\bar{\rho}$.

When applying this formula, it is possible to solve two problems:

- the direct problem of orientation of a rigid body, when a change in the position of the body in space is found for a known rotation vector;

- an inverse problem, in which the vector of the final rotation is determined at the known value of its modulus for the known initial and final positions of the body.

While the direct problem is often solved in analytical mechanics, solving the inverse problem, when the Rodrigues formula is considered as an equation with respect to $\theta^{-}$,commonly used codes do not provide any solution. For example, Mathcad does not find an analytical solution in symbolic form. Maxima was unable to find an analytical solution in four and a half hours, and eventually stopped itself. After a day of analytical calculations, Maple receives a solution on forty pages, and from the point of view of mathematics, such a solution is too long to be used in practice as shown in Table 1.

Table 1. Results of solving the Rodrigues equation by software packages

\begin{tabular}{|c|c|c|}
\hline & $\begin{array}{c}\text { Software } \\
\text { Application } \\
\text { package }\end{array}$ & Solution or problems \\
\hline & Maxima & $\begin{array}{c}\text { Analytical solution in symbolic } \\
\text { form not found } \\
\text { analytical solution - solution not } \\
\text { found }\end{array}$ \\
\hline & Maple & $\begin{array}{c}25 \text { hours - analytical solution in } \\
\text { the form of } 40 \text { pages of text (the } \\
\text { solution is boundless) }\end{array}$ \\
\hline
\end{tabular}

Therefore, the authors have proposed their own analytical solution, which is considered below.

\section{Solution of the Rodrigues equation}

Let's write down the Rodrigues formula:

$$
\overline{\rho^{\prime}}=\bar{\rho}+\frac{1}{1+\frac{1}{4}|\bar{\theta}|^{2}} \bar{\theta} \times\left(\bar{\rho}+\frac{1}{2} \bar{\theta} \times \bar{\rho}\right)
$$

Let us assume that the vectors $\overline{\rho^{\prime}}$ and $\bar{\rho}$ are known (for example, given by the results of an experiment).

It is required to find the rotation vector $\bar{\theta}$ with its known modulus $|\bar{\theta}|$.

In Fig. 2. shows an illustration of the vectors involved in the Rodrigues formula. 


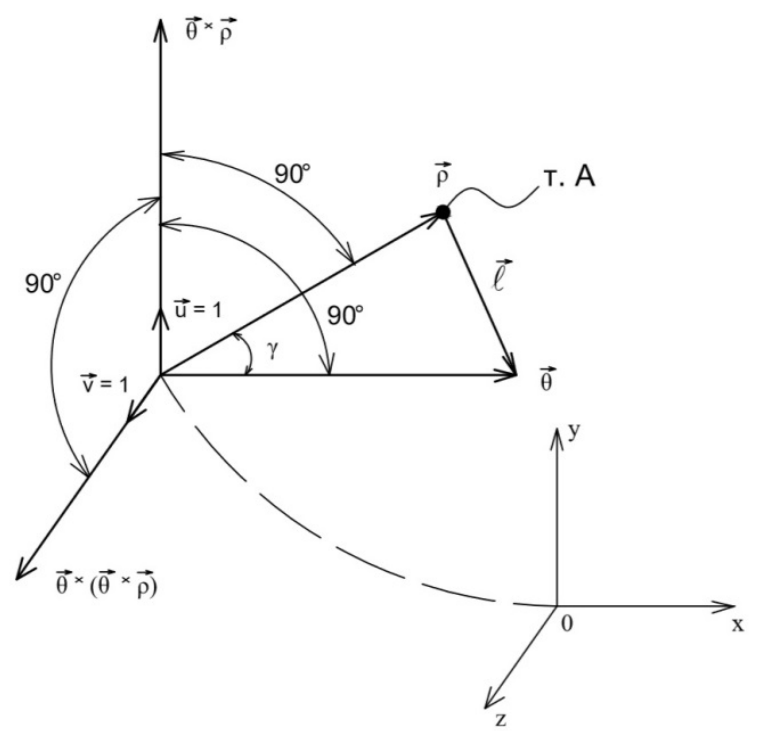

Fig. 2. Rodrigues' rotation formula illustration.

Let be

$$
\begin{gathered}
\alpha=1+\frac{1}{4}|\bar{\theta}|^{2} \\
\mathrm{x}_{\mathrm{A}}=\rho_{1}, \mathrm{y}_{\mathrm{A}}=\rho_{2}, z_{\mathrm{A}}=\rho_{3}
\end{gathered}
$$

Let's rewrite formula (1) as:

$$
2 \alpha \overline{\Delta \rho}=2 \bar{\theta} \times \bar{\rho}+\bar{\theta} \times(\bar{\theta} \times \bar{\rho}),
$$

where

$$
\overline{\Delta \rho}=\overline{\rho^{\prime}}-\bar{\rho}=\bar{r}
$$

and further we will consider it as an equation with $\bar{\theta}$. In this case, the projections of the vectors on the axes of the coordinate system $O x y z$ will be accompanied by indices $1,2,3$, and the unit vectors of the axes $O x, O y, O z$ will be denoted $\bar{l}, \bar{\jmath}, \bar{k}$, respectively.

Multiplying scalarly (2) by, we obtain equation (4):

$$
2 \alpha(\bar{r}, \bar{\theta})=0+0=0 \Rightarrow r_{1} \theta_{1}+r_{2} \theta_{2}+r_{3} \theta_{3}=0
$$

Then we notice the fulfillment of the following equalities:

$$
2 \alpha(\bar{r}, \bar{\rho})=(\bar{\theta} \times(\bar{\theta} \times \bar{\rho}), \bar{\rho})
$$

Next, we notice that the following equalities are fulfilled:

$$
\begin{gathered}
\bar{\theta} \times \bar{\rho}=|\bar{\theta}||\bar{\rho}| \sin \gamma \cdot \bar{u} \\
\bar{\theta} \times(\bar{\theta} \times \bar{\rho})=|\bar{\theta}|^{2}|\bar{\rho}| \cdot \sin \gamma \cdot \bar{v} \\
(\bar{\theta} \times(\bar{\theta} \times \bar{\rho}), \bar{\rho})=|\bar{\theta}|^{2}|\bar{\rho}|^{2} \sin \gamma \cdot \cos \left(90^{\circ}+\gamma\right) \\
=-|\bar{\theta}|^{2}|\rho|^{2} \sin ^{2} \gamma
\end{gathered}
$$

From here we get:

$$
\sin ^{2} \gamma=-2 \alpha \frac{(\bar{r}, \bar{\rho})}{|\bar{\theta}|^{2}|\bar{\rho}|^{2}}, \cos ^{2} \gamma=1+2 \alpha \frac{(\bar{r}, \bar{\rho})}{|\theta|^{2}|\rho|^{2}}
$$

and, therefore:

$$
\cos \gamma=\sqrt{\frac{|\bar{\theta}|^{2}|\bar{\rho}|^{2}+2 \alpha(\bar{r}, \bar{\rho})}{|\bar{\theta}|^{2}|\bar{\rho}|^{2}}}
$$

Now we will find an analytical expression for equation (4), sequentially calculating vector result $\bar{\theta} \times \bar{\rho}$ multiplication and further $\bar{\theta} \times(\bar{\theta} \times \bar{\rho})$.. Performed:

$$
\begin{gathered}
\bar{\theta} \times \bar{\rho}=\bar{l}\left(\theta_{2} \rho_{3}-\rho_{2} \theta_{3}\right)+\bar{\jmath}\left(-\theta_{1} \rho_{3}+\rho_{1} \theta_{3}\right) \\
+\bar{k}\left(\theta_{1} \rho_{2}-\rho_{1} \theta_{2}\right)
\end{gathered}
$$

Let's introduce a change of variables:

$$
\begin{gathered}
\theta_{1}^{\prime}=\theta_{2} \rho_{3}-\rho_{2} \theta_{3} \\
\theta_{2}^{\prime}=-\theta_{1} \rho_{3}+\rho_{1} \theta_{3} \\
\theta_{3}^{\prime}=\theta_{1} \rho_{2}-\rho_{1} \theta_{2}
\end{gathered}
$$

Using the new variables, let's write an expression for: $\bar{\theta} \times(\bar{\theta} \times \bar{\rho})$ :

$$
\bar{\theta} \times(\bar{\theta} \times \bar{\rho})=\left|\begin{array}{ccc}
\overline{\mathrm{l}} & \overline{\mathrm{j}} & \overline{\mathrm{k}} \\
\theta_{1} & \theta_{2} & \theta_{3} \\
\theta_{1}^{\prime} & \theta_{2}^{\prime} & \theta_{3}^{\prime}
\end{array}\right|=\overline{\mathrm{i}}\left(\theta_{2} \theta_{3}^{\prime}-\theta_{2}^{\prime} \theta_{3}\right)+
$$

$\overline{\mathrm{j}}\left(-\theta_{1} \theta_{3}^{\prime}+\theta_{1}^{\prime} \theta_{3}\right)+\overline{\mathrm{k}}\left(\theta_{1} \theta_{2}^{\prime}-\theta_{1}^{\prime} \theta_{2}\right)=\mathrm{i}\left[\theta_{2}\left(\theta_{1} \rho_{2}-\right.\right.$ $\left.\left.\rho_{1} \theta_{2}\right)-\theta_{3}\left(\rho_{1} \theta_{3}-\rho_{3} \theta_{1}\right)\right]+\bar{j}\left[\theta_{3}\left(\theta_{2} \rho_{3}-\rho_{2} \theta_{3}\right)-\right.$ $\left.\theta_{1}\left(\theta_{1} \rho_{2}-\rho_{1} \theta_{2}\right)\right]+\bar{k}\left[\theta_{1}\left(\rho_{1} \theta_{3}-\rho_{3} \theta_{1}\right)-\right.$ $\left.\theta_{2}\left(\theta_{2} \rho_{3}-\rho_{2} \theta_{3}\right)\right]=\overline{1}\left(\rho_{2} \theta_{1} \theta_{2}-\rho_{1} \theta_{2}^{2}-\rho_{1} \theta_{3}^{2}+\right.$ $\left.\rho_{3} \theta_{1} \theta_{2}\right)+\overline{\mathrm{j}}\left(\rho_{3} \theta_{2} \theta_{3}-\rho_{2} \theta_{3}^{2}-\rho_{2} \theta_{1}^{2}+\rho_{1} \theta_{1} \theta_{2}\right)+$ $\bar{\kappa}\left(\rho_{1} \theta_{1} \theta_{3}-\rho_{3} \theta_{1}^{2}-\rho_{3} \theta_{2}^{2}+\rho_{2} \theta_{2} \theta_{3}\right)$

From here we find

$(\bar{\theta} \times(\bar{\theta} \times \bar{\rho}), \bar{\rho})=\rho_{1} \rho_{2} \theta_{1} \theta_{2}-\rho_{1}^{2} \theta_{2}^{2}-\rho_{1}^{2} \theta_{3}^{2}+$ $\rho_{1} \rho_{3} \theta_{1} \theta_{3}++\rho_{2} \rho_{3} \theta_{2} \theta_{3}-\rho_{2}^{2} \theta_{3}^{2}-\rho_{2}^{2} \theta_{1}^{2}+\rho_{1} \rho_{2} \theta_{1} \theta_{2}+$ $\rho_{1} \rho_{3} \theta_{1} \theta_{3}-\rho_{3}^{2} \theta_{1}^{2}-\rho_{3}^{2} \theta_{2}^{2}+\rho_{3} \rho_{2} \theta_{2} \theta_{3}==-\left(\rho_{1} \theta_{2}-\right.$ $\left.\rho_{2} \theta_{1}\right)^{2}-\left(\rho_{1} \theta_{3}-\rho_{3} \theta_{1}\right)^{2}-\left(\rho_{2} \theta_{3}-\rho_{3} \theta_{2}\right)^{2}$

Therefore, equation (4) is obtained in the form (6):

$$
\begin{gathered}
\left(\rho_{1} \theta_{2}-\rho_{2} \theta_{1}\right)^{2}+\left(\rho_{1} \theta_{3}-\rho_{3} \theta_{1}\right)^{2}+ \\
\left(\rho_{2} \theta_{3}-\rho_{3} \theta_{2}\right)^{2}=-2 \alpha(\bar{r}, \bar{\rho})
\end{gathered}
$$

In this case, the following equalities are fulfilled:

$$
\left\{\begin{array}{c}
\theta_{1}^{2}+\theta_{2}^{2}+\theta_{3}^{2}=|\bar{\theta}|^{2} \\
\left(\theta_{1}-\rho_{1}\right)^{2}+\left(\theta_{2}-\rho_{2}\right)^{2}+\left(\theta_{3}-\rho_{3}\right)^{2}=\ell^{2}
\end{array}\right.
$$

where $|\bar{\theta}|^{2}-$ a known value equal to $4 \operatorname{tg}^{2} \frac{\chi}{2}$, where is known $\chi$ - angle of rotation of the vector $\bar{\rho}$ Expanding the brackets, we get:

$$
\begin{gathered}
\theta_{1}^{2}-2\left(\theta_{1} \rho_{1}+\theta_{2} \rho_{2}+\theta_{3} \rho_{3}\right)+\theta_{2}^{2}+\theta_{3}^{2}+\rho_{1}^{2}+\rho_{2}^{2} \\
+\rho_{3}^{2}=\ell^{2}
\end{gathered}
$$

or

$$
\theta_{1} \rho_{1}+\theta_{2} \rho_{2}+\theta_{3} \rho_{3}=\frac{|\bar{\rho}|^{2}+|\bar{\theta}|^{2}-\ell^{2}}{2}=c
$$


Since $\ell^{2}=|\bar{\rho}|^{2}+|\bar{\theta}|^{2}-2|\bar{\rho}||\bar{\theta}| \cos \gamma$, by virtue of formula (5) we arrive at the expression:

$$
c=|\bar{\rho}||\bar{\theta}| \cos \gamma=\sqrt{|\bar{\theta}|^{2}|\bar{\rho}|^{2}+2 \alpha(\bar{r}, \bar{\rho})}
$$

Equation (7) is combined with equation (3):

$$
\left\{\begin{array}{l}
\theta_{1} \rho_{1}+\theta_{2} \rho_{2}+\theta_{3} \rho_{3}=c \\
r_{1} \theta_{1}+r_{2} \theta_{2}+r_{3} \theta_{3}=0
\end{array}\right.
$$

Now, from (8), we express in terms $\theta_{3}$ of the quantities $\theta_{1}, \theta_{2}$ :

$$
\begin{aligned}
& \theta_{1}=\frac{r_{2} c}{r_{2} \rho_{1}-r_{1} \rho_{2}}+\frac{r_{3} \rho_{2}-r_{2} \rho_{3}}{r_{2} \rho_{1}-r_{1} \rho_{2}} \theta_{3}=a_{1}+b_{1} \theta_{3} \\
& \theta_{2}=\frac{r_{1} c}{r_{1} \rho_{2}-r_{2} \rho_{1}}+\frac{r_{3} \rho_{1}-r_{1} \rho_{3}}{r_{1} \rho_{2}-r_{2} \rho_{1}} \theta_{3}=a_{2}+b_{2} \theta_{3}
\end{aligned}
$$

Substituting these values into equation (6), we get:

$$
\begin{gathered}
\left(\rho_{1}\left(a_{2}+b_{2} \theta_{3}\right)-\rho_{2}\left(a_{1}+b_{1} \theta_{3}\right)\right)^{2} \\
+\left(\rho_{1} \theta_{3}-\rho_{3}\left(a_{1}+b_{1} \theta_{3}\right)\right)^{2}+ \\
+\left(\rho_{2} \theta_{3}-\rho_{3}\left(a_{2}+b_{2} \theta_{3}\right)\right)^{2}=-2 \alpha(\bar{r}, \bar{\rho})^{2}
\end{gathered}
$$

Let us introduce the notation.

$$
\rho_{13}=\rho_{1}^{2}+\rho_{3}^{2} ; \rho_{23}=\rho_{2}^{2}+\rho_{3}^{2} ; \rho_{12}=\rho_{1}^{2}+\rho_{2}^{2}
$$

After reducing the homogeneous terms in (10), we arrive at the equation:

$$
\begin{gathered}
\left(\rho_{13} b_{2}^{2}+\rho_{23} b_{1}^{2}+\rho_{12}-2 \rho_{1} \rho_{2} b_{1} b_{2}-2 \rho_{1} \rho_{3} b_{1}\right. \\
\left.-2 \rho_{2} \rho_{3} b_{2}\right) \theta_{3}^{2}+ \\
+\left(2 \rho_{13} a_{2} b_{2}+2 \rho_{23} a_{1} b_{1}-2 \rho_{1} \rho_{2}\left(b_{2} a_{1}+b_{1} a_{2}\right)\right. \\
\left.-2 \rho_{1} \rho_{3} a_{1}-2 \rho_{2} \rho_{3} a_{2}\right) \theta_{3}+ \\
+\rho_{13} a_{2}^{2}+\rho_{23} a_{1}^{2}-2 \rho_{1} \rho_{2} a_{2} a_{1}=-2 \alpha(\bar{r}, \bar{\rho})
\end{gathered}
$$

Notate:

$$
\begin{gathered}
\rho_{13} b_{2}^{2}+\rho_{23} b_{1}^{2}+\rho_{12}-2 \rho_{1} \rho_{2} b_{1} b_{2}-2 \rho_{1} \rho_{3} b_{1} \\
-2 \rho_{2} \rho_{3} b_{2}=L_{2} \\
2 \rho_{13} a_{2} b_{2}+2 \rho_{23} a_{1} b_{1}-2 \rho_{1} \rho_{2}\left(b_{2} a_{1}+b_{1} a_{2}\right) \\
-2 \rho_{1} \rho_{3} a_{1}-2 \rho_{2} \rho_{3} a_{2}=L_{1} \\
\rho_{13} a_{2}^{2}+\rho_{23} a_{1}^{2}-2 \rho_{1} \rho_{2} a_{2} a_{1}=L_{o}
\end{gathered}
$$

the solution with respect to $\theta_{3}$, assuming the angle $\gamma$ to be acute, is obtained in the form:

$$
\theta_{3}=\frac{-\mathrm{L}_{1}+\sqrt{\mathrm{L}_{1}^{2}-4 \mathrm{~L}_{2}\left(\mathrm{~L}_{\mathrm{o}}+2 \alpha(\overline{\mathrm{r}}, \bar{\rho})\right)}}{2 \mathrm{~L}_{2}}
$$

After that, using formulas (9), we find the values of $\theta_{1}$ and $\theta_{2}$. Vector $\theta$ found. Dividing by $\bar{\theta}|\bar{\theta}|$, we find the unit vector of the rotation axis $\bar{e}$.

\section{Validation of the solution in the Maple software package}

To validate the solution, the authors used the inverse method implemented in Maple. For the a priori given components of the vectors $\theta^{-}$and $\rho$, the algorithm described above was checked for validity. According to the results of repeated modeling, the components of the vector $\theta^{-}$certainly agreed with those specified with an accuracy of up to the fourth decimal place, with which the calculations themselves were carried out. In this case, pairs of roots were obtained, which was due to the solution of this equation, depending on the value of the angle $\gamma$, acute or obtuse.

\section{Conclusion}

In this paper, the Rodrigues equation for determining the volumetric accuracy of multi-axis CNC-controlled systems has been solved. An algorithm for calculating the position of the axis of a rotary kinematic pair in problems of volumetric accuracy of mechanical motion of a portal-type system with an additional pair of rotation was proposed based on the analytical solution of the the inverse problem of finding the vector of the final rotation of the known modulus from the known initial and final values of the characteristic vector of the rotated rigid body. The obtained results make it possible to expand the dimension of the space of generalized coordinates of the system analyzed for the volumetric accuracy from three to four that can be used as the basis for achieving the dimension of the universal generalized space of a controlled mechanical system, while reducing amount of probabilistic estimates related to the problem of the volumetric accuracy. This is expected to make an important contribution to the development of ultra-precise systems of controlled mechanical movement . The analytical results obtained in this work were verified by comparing with the numerical solutions of the inverse problem in Maple.

\section{References}

1. S.N. Grigoriev, D.A. Masterenko, V.I. Teleshevskii., P.N. Emelyanov, Measurement Techniques, 55(11), 1311-1315 (2013)

2. V.I. Teleshevskii, V.A. Sokolov, Y.I. Pimushkin, Journal of Physics: Conference Series, 998(1), 012034 (2018)

3. V.I. Teleshevskii, V.A. Sokolov Y.I. Pimushkin, Journal of Physics: Conference Series, 1260(3), 032038 (2019)

4. P.A. McKeown, J. Loxham, Ann. CIRP, 22(1) (1973)

5. H. Schwenke et. al., Ann. CIRP, 57, 660-675 (2008)

6. H. Schwenke, M. Franke, J. Hannaford, Ann. CIRP, 54(1), 475-478 (2005)

7. N. A. Serkov, Vestnik of Scientific and Technical Development, 3(55), 26-46 (2012) 
8. L.N. Lamikiz, López de Lacalle, O. Ocerin, D. Díez, E. Maidagan, International Journal of Advanced Manufacturing Technology, 37, C. 122-139

9. M. Rahman, J. Heikkala, K. Lappalainen, International Journal of Machine Tools \& Manufacture, 40, 1535-1546 (2000)

10. A.C. Okafor, Y.M. Ertekin, International Journal of Machine Tools \& Manufacture, 40(8), 1199-1213 (2000)

11. Y. Lin, Y. Shen, International Journal of Advanced Manufacturing Technology, 21, 243-248 (2003)

12. K.Gee. Ahn, D.W. Cho, International Journal of Machine Tools \& Manufacture, 40, 2235-2248 (2000)

13. Q. Cheng, Q. Feng, Z. Liu, P. Gu, L. Cai, Proceedings of the Institution of Mechanical Engineers, Part C: Journal of Mechanical Engineering Science, 229, 2534-2550 (2015) $10.1177 / 0954406214562633$ 